\title{
Andrographolide inhibits growth of human T-cell acute lymphoblastic leukemia Jurkat cells by downregulation of PI3K/AKT and upregulation of p38 MAPK pathways
}

\author{
This article was published in the following Dove Press journal: \\ Drug Design, Development and Therapy \\ II April 2016 \\ Number of times this article has been viewed
}

\author{
Tingfang Yang' \\ Shuluan $\mathrm{Yao}^{2}$ \\ Xianfeng Zhang ${ }^{3}$ \\ Yan Guo ${ }^{2}$ \\ 'Department of Pediatrics, Jining \\ No I People's Hospital, Shandong \\ Province, People's Republic of China; \\ ${ }^{2}$ Department of Respiratory Medicine, \\ Jining Medical University Affiliated \\ Hospital, Shandong Province, People's \\ Republic of China; ${ }^{3}$ Department of \\ Psychiatry, Jining Psychiatric Hospital, \\ Shandong Province, People's Republic \\ of China
}

\begin{abstract}
T-cell acute lymphoblastic leukemia (T-ALL) as a prevalent hematologic malignancy is one of the most common malignant tumors worldwide in children. Andrographolide (Andro), the major active component from Andrographis paniculata, has been shown to possess antitumor activities in several types of cancer cells. However, whether Andro would inhibit T-ALL cell growth remains unclear. In this study, we investigated the cytotoxic effect of Andro on human T-ALL Jurkat cells and explored the mechanisms of cell death. Cell apoptosis was assayed by flow cytometry, and the signaling transduction for Andro was analyzed by Western blotting. The results indicated $10 \mu \mathrm{g} / \mathrm{mL}$ Andro could significantly induce Jurkat cells' apoptosis, depending on the inhibition of PI3K/AKT pathway. Moreover, Andro-induced apoptosis is enhanced by AKT-selective inhibitor LY294002. ERK- or JNK-selective inhibitors PD98059 and SP600125 had no effect on Andro-induced apoptosis. In addition, p38 inhibitor SB203580 could reverse Andro-induced apoptosis in Jurkat cells. We also found that the protein expression of p-p53 and p-p38 were increased after Andro treatments. The result of an in vivo study also demonstrated Andro's dose-dependent inhibition in subcutaneous Jurkat xenografts. In conclusion, our findings explained a novel mechanism of drug action by Andro in Jurkat cells and suggested that Andro might be developed into a new candidate therapy for T-ALL patients in the coming days.
\end{abstract}

Keywords: andrographolide, PI3K, AKT, Burkitt lymphoma, Jurkat cell

\section{Background}

T-cell acute lymphoblastic leukemia (T-ALL) are aggressive proliferations of transformed T-cell progenitors, which accounts for $10 \%-15 \%$ of T-ALL cases in children and $25 \%$ of adult T-ALL cases. ${ }^{1}$ Conventional cancer therapies could lead to a complete remission rate of $85 \%$ and a high cure rate in childhood T-ALL, but adult T-ALL patients are at increased risk of serious adverse effects. ${ }^{2,3}$ The prognosis for relapsing patients is poor, with only $15 \%-25 \%$ achieving stable remission after second-line treatment, ${ }^{1-3}$ and the 5-year survival rate for adult T-ALL patients is as low as $45 \%-55 \%$. These outcomes underscore the need to develop more effective therapies to treat T-ALL patients.

Phosphoinositide 3-kinases (PI3K) play crucial roles in many cellular processes, including cell proliferation, differentiation, survival, angiogenesis, and so on. ${ }^{4,5} \mathrm{PI} 3 \mathrm{~K}$ mediates signals from receptor tyrosine kinases, followed by phosphorylating protein kinase B, also known as AKT. AKT phosphorylation subsequently activates mammalian target of rapamycin and downstream molecules, such as $70 \mathrm{kd} \mathrm{S6}$ protein kinases (P70S6K) and
Correspondence: Tingfang Yang Department of Pediatrics, Jining No I People's Hospital, 6 Jiankang Road, Jining City, Shandong Province 2720I I, People's Republic of China

Tel +865372253104

Email ytfang88@126.com 
eukaryotic translation initiation factor 4E-binding protein 1, consequently regulating cell growth and gene transcription. ${ }^{6}$

Andrographolide (Andro) is a bioactive diterpenoid isolated from a traditional herbal medicine named Andrographis paniculata, which is commonly used in Southeast Asian countries. ${ }^{7,8}$ Previous studies have proved that Andro possesses wide pharmacological activities, including anticancer, antioxidative, anti-inflammatory, and antiviral effects. ${ }^{9-11}$ Regarding anticancer activity, Andro exerts its effect on tumor cells through several mechanisms, such as cellular migration, growth factor signaling transduction, cell-cycle arrest, and angiogenesis. ${ }^{12-14}$ For example, Andro could induce human leukemic HL-60 cell apoptosis through mitochondrial-mediated pathway. ${ }^{15}$ Andro is also able to inhibit human colorectal carcinoma Lovo cell migration and invasion due to the downregulation of matrix metalloproteinase-7 expression. ${ }^{14}$ However, no systematic study has been reported regarding anticancer effect of Andro on T-ALL cells. Here, we report the cell death in T-ALL Jurkat cells induced by Andro and investigate the underlying mechanisms from molecular perspectives.

\section{Materials and methods Cell growth inhibition assay}

T-ALL Jurkat cells were purchased from American Type Culture Collection (Manassas, VA, USA). The cells were cultured in Dulbecco's Modified Eagle's Medium (DMEM) supplemented with $10 \%$ fetal bovine serum, $10^{5} \mathrm{U} / \mathrm{L}$ penicillin, and $100 \mathrm{mg} / \mathrm{L}$ streptomycin (Thermo Fisher Scientific, Waltham, $\mathrm{MA}, \mathrm{USA}$ ) at $37^{\circ} \mathrm{C}$ in an atmosphere containing $5 \% \mathrm{CO}_{2}$. The cells were dispensed in 96-well, flat bottom microtiter plates (NUNC, Roskilde, Denmark) at a density of $1.0 \times 10^{4}$ cells per well. After 24 hours (h) incubation, the cells were treated with different concentrations of Andro $(0-32 \mu \mathrm{g} / \mathrm{mL})$ for the indicated time periods. Then, a $20 \mu \mathrm{L}$ aliquot of MTT solution $(5.0 \mathrm{mg} / \mathrm{mL})$ was added to each well, followed by $4 \mathrm{~h}$ incubation, and then an enzyme-linked immunosorbent assay (ELISA) reader (Thermo Fisher Scientific, Waltham, MA USA) was used to measure the optical density. Andro was purchased from Sigma-Aldrich Co., (St Louis, MO, USA), with a purity of $\geq 95 \%$ high performance liquid chromatography (HPLC). The study was approved by the ethics committee of Jining No 1 People's Hospital.

\section{Apoptosis assay}

Annexin V-fluorescein isothiocyanate(FITC)/propidium iodide (PI) double-staining method was used to analyze apoptotic cells. Briefly, Jurkat cells were treated with different concentrations of Andro for $48 \mathrm{~h}$. Cells were digested into single-cell suspension with ethylenediaminetetraacetic acid (EDTA)-free trypsin and stained according to the manufucturer's instructions provided with the Annexin V-FITC/PI Apoptosis Detection kit (Pierce Biotechnology, Rockford, IL, USA). After that, stained cells were analyzed by flow cytometry (BD, Franklin Lake, NJ, USA) as soon as possible.

\section{Autophagy detection}

Monodansylcadaverine (MDC) is a specific marker for autophagic vacuoles. ${ }^{16}$ To confirm that Andro treatments could induce Jurkat cells autophagy, we labeled autophagic vacuoles with MDC. The autophagic vacuoles were labeled with MDC by incubating with $0.05 \mathrm{mM} / \mathrm{L} \mathrm{MDC}$ in phosphate-buffered saline (PBS) at $37^{\circ} \mathrm{C}$ for $2 \mathrm{~h}$. Then, cells were washed three times with cold PBS buffer and immediately detected using flow cytometry.

\section{Western blotting analysis}

Western blot was performed as described previously with some modifications. ${ }^{17}$ The cells were washed with $1 \times$ PBS and harvested on ice. Lysates were prepared in a Western lysis buffer (20 mM Tris [pH 7.5], $150 \mathrm{mM} \mathrm{NaCl,} \mathrm{1 \%} \mathrm{Triton}$ $\mathrm{X}-100$, sodium pyrophosphate, $\beta$-glycerophosphate, EDTA, $\mathrm{Na}_{3} \mathrm{VO}_{4}$, and leupeptin) by repeatedly passing cells through a pipette. Protein concentration was determined using the BCA protein assay kit (Promega, Madison, WI, USA). About $30 \mu \mathrm{g}$ total protein was separated by electrophoresis on $10 \%-12 \%$ standard sodium dodecyl sulfate-polyacrylamide gels and then transferred to nitrocellulose membranes (Bio-Rad Laboratories, Hercules, CA, USA). After blocking with 5\% nonfat dry milk in Tris-buffered saline containing $0.05 \%$ Tween-20, the membrane was incubated with the primary antibody (1:1,000 dilution) overnight at $4^{\circ} \mathrm{C}$. After three washes, the membrane was incubated with horseradish peroxidaseconjugated secondary antibodies (1:2,000 dilutions) for $1 \mathrm{~h}$. Then, the immunoreactive protein bands were visualized by using the Enhanced Chemiluminescence kit (GE Healthcare, Buckinghamshire, UK). All antibodies were purchased from Cell Signaling Technology (Danvers, MA, USA).

\section{Real-time polymerase chain reaction (PCR) analysis}

Jurkat cells were treated with different concentrations of Andro for $48 \mathrm{~h}$. Then, cells were harvested and RNA was extracted from lysates using Qiagen RNA easy kits (QIAGEN Inc., Valencia, CA, USA). We verified RNA quality by running samples on an Agilent Bioanalyzer 2100 (Agilent Technologies, Inc., Santa Clara, CA, USA). Then, $1 \mu \mathrm{g}$ of RNA was digested with DNase and reverse transcribed with random primers and Im-Prom II reverse transcriptase (Promega, Madison, WI, USA). Later, 
the cDNA was subjected to RT-PCR using specific primers for $A K T, p 38, J N K, E R K$, and GAPHD. Quantitative RT-PCR was performed in a $25 \mu \mathrm{L}$ reaction mixture using SYBR Green IQ supermix (Bio-Rad Laboratories, Hercules, CA, USA) according to the manufacturer's instructions. Gene expression levels were normalized to GAPHD.

\section{In vivo study}

Nude mice were purchased from Shanghai SLAC Laboratory Animal Co., Ltd. (Shanghai, China), and were inoculated with $3 \times 10^{6}$ Jurkat cells subcutaneously in the right and left flanks. Tumor sizes were calculated by the standard formula of Tumor size $=$ Length $\times$ Width $\times$ Width $/ 2$. Body weights and tumor weights were measured using a balance. When the tumor size of mice reached $150-180 \mathrm{~mm}^{3}$, they were randomly divided into four groups. One group was treated with PBS as vehicle control; the other groups were treated with 5, 10, and $20 \mathrm{mg} / \mathrm{kg}$ Andro, respectively. Animals were killed 3 weeks after initial injections, and a final measurement of tumor volume and weight were taken using a caliper and a balance (Mettler Toledo, Schwerzenbach, Switzerland), respectively. All animal experiments were performed in accordance with protocols approved by the Jining No 1 People's Hospital Experimental Animal Care and Use Committee.

\section{Statistical analysis of the data}

Statistical comparisons were made using Student's $t$-test, twoway comparisons by statistical package for social sciences (SPSS) version 12.00 (SPSS Inc., Chicago, IL, USA). A level of significance of $P<0.05$ was utilized, and the data were presented as mean \pm standard deviation (SD) for the indicated number of independently performed experiments.

\section{Results}

\section{Andro inhibited growth of Jurkat cells}

To identify the therapeutic potentials of Andro, T-ALL Jurkat cells were cultured with different concentrations of Andro for 24,48 , and $72 \mathrm{~h}$, and then cell viability was determined by 3-(4,5-dimethyl-2-thiazolyl)-2,5-diphenyl-2-H-tetrazolium bromide (MTT) assay. The results showed that Andro inhibited the growth of Jurkat cells in a dose- and time-dependent manner: $50 \%$ inhibition $\left(\mathrm{IC}_{50}\right)$ at 24,48 , and $72 \mathrm{~h}$ was observed at $18.5,9.3$, and $6.5 \mu \mathrm{g} / \mathrm{mL}$, respectively (Figure 1).

\section{Andro-inhibited growth of Jurkat cells depended on apoptosis}

Since Andro could induce apoptosis in some other cancer cells, ${ }^{18,19}$ we intended to investigate whether Andro could induce apoptosis in Jurkat cells by Annexin V-FITC/PI

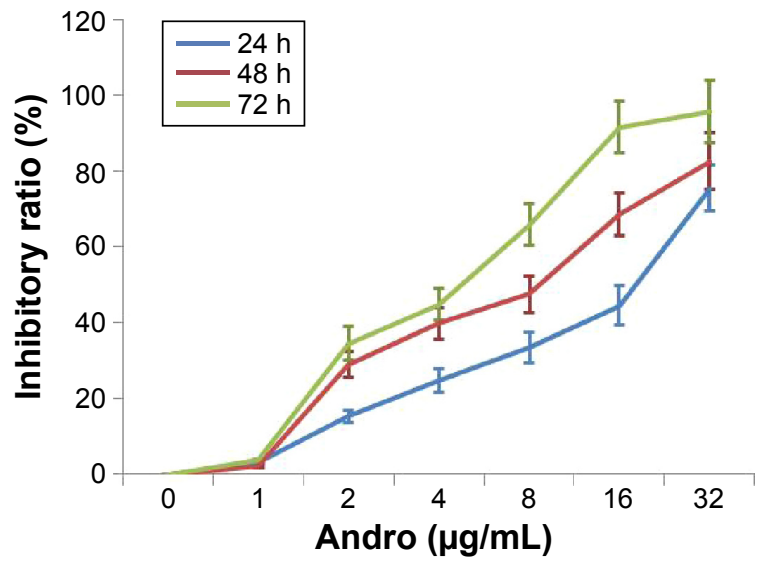

Figure I Antiproliferation effect of Andro on Jurkat cells.

Notes: Jurkat cells were cultured in 96-well, flat bottom microtiter plates at a density of $1.0 \times 10^{4}$ cells per well for 24 hours. Then, the cells were treated with different concentrations of Andro for 24, 48, and 72 hours, and then cell viability was determined by MTT assay. Mean $\pm S D, n=3$.

Abbreviations: Andro, Andrographolide; SD, standard deviation; MTT, 3-(4,5dimethyl-2-thiazolyl)-2,5-diphenyl-2-H-tetrazolium bromide.

double-staining method. As shown in Figure 2A and B, Andro significantly induced Jurkat cell apoptosis after $48 \mathrm{~h}$ treatment. This finding was further confirmed by morphological observation of the cells. Plenty of small apoptosis bodies were observed after Andro treatment (Figure 2C). The apoptosis induced by Andro was significantly reduced when the cells were treated with pan-caspase inhibitor z-VAD (Figure 2A). The activated caspase-3 protein expression levels were confirmed by performing a Western blot analysis in Jurkat cells treated with z-VAD (Figure 2D).

\section{Andro-induced Jurkat cell growth inhibition was independent of autophagy}

Many reports have already indicated the importance of autophagy in tumor suppression, which is known as a response to various anticancer therapies in many kinds of cancer cells. ${ }^{20}$ To study whether autophagy is involved in Andro-induced growth inhibition of Jurkat cells, MDC staining was first performed to detect the autophagic vacuoles. The result showed that there was no MDC recruitment to autophagosomes induced by Andro in Jurkat cells (Figure 3A). This result was confirmed by the nonconversion of cytosolic LC3-I to lipidated, autophagosome membrane-bound LC3-II, a specific marker for autophagosome formation (Figure 3B). Taken together, Andro-inhibited growth of Jurkat cells was independent of autophagy.

\section{Andro-inhibited growth of Jurkat cells depended on PI3K/AKT downregulation and p38 upregulation}

Recent studies reported that PI3K/AKT, ERK, p38, and JNK were involved in Andro-induced cancer cell inhibition. ${ }^{21,22}$ 
A

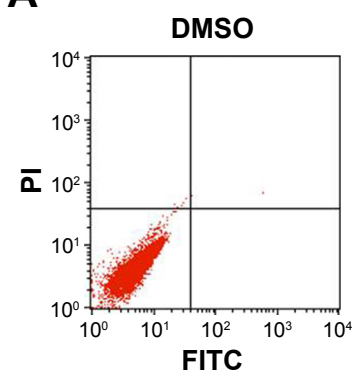

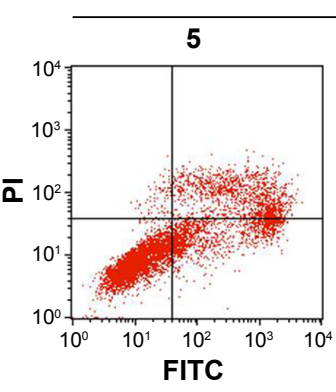

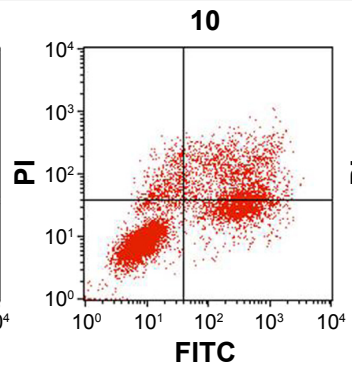

Andro $(\mu \mathrm{g} / \mathrm{mL})$

B
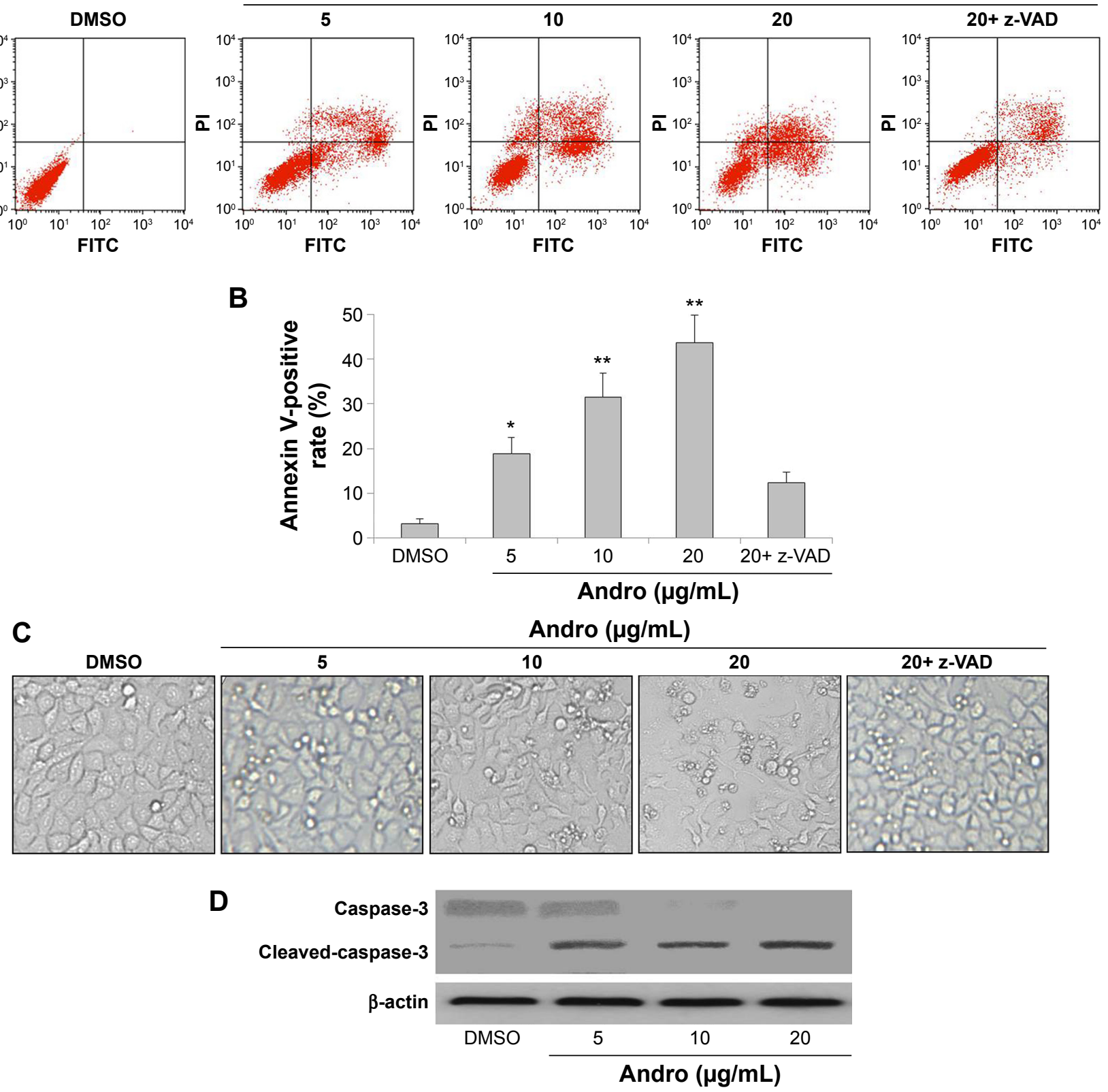

Figure 2 Andro induced apoptosis in Jurkat cells.

Notes: (A and B) Jurkat cells were treated with different concentrations of Andro and/or z-VAD for 48 hours. Cells were digested to form a single-cell suspension with EDTAfree trypsin and stained according to the manufacturer's instruction provided with the Annexin V-FITC/PI Apoptosis Detection kit. After that, stained cells were analyzed by flow cytometry. (C) Jurkat cells cultured in the media were observed under inverted microscope. (D) Jurkat cells were washed with I $\times$ PBS and harvested on ice. Lysates were prepared using a lysis buffer and then subjected to Western blot analysis to detect capase-3 protein expression. Mean $\pm S D, n=3$. $* P<0.05$, $* * P<0.0$ I versus $D M S O$ group. Abbreviations: Andro, Andrographolide; EDTA, ethylenediaminetetraacetic acid; FITC, fluorescein isothiocyanate; PI, propidium iodide; DMSO, dimethyl sulfoxide; SD, standard deviation; PBS, phosphate-buffered saline.

Therefore, we next investigated the effects of Andro on the PI3K/AKT, p53, ERK, JNK, and p38 pathways in Jurkat cells. As shown in Figure 4A, $10 \mu \mathrm{g} / \mathrm{mL}$ Andro could significantly inhibit p-AKT expression in Jurkat cells. In addition, the expressions of p-ERK and p-JNK were not affected by Andro. Moreover, we found that p-p53 and p-p38 protein expression were upregulated after Andro treatments. More interestingly, the RNA level of $p 38$ was also upregulated (Figure 4B). To further determine the roles of AKT, ERK, and p38 in Andro-induced Jurkat cell inhibition, selective inhibitors specific to them were used. As illustrated in Figure 4C, Andro-induced cell growth inhibition is enhanced by AKT-selective inhibitor LY294002 but not ERK-selective inhibitor PD98059 or JNK inhibitor SP600125. Moreover, p38 inhibitor SB203580 could reverse Andro-induced growth inhibition 


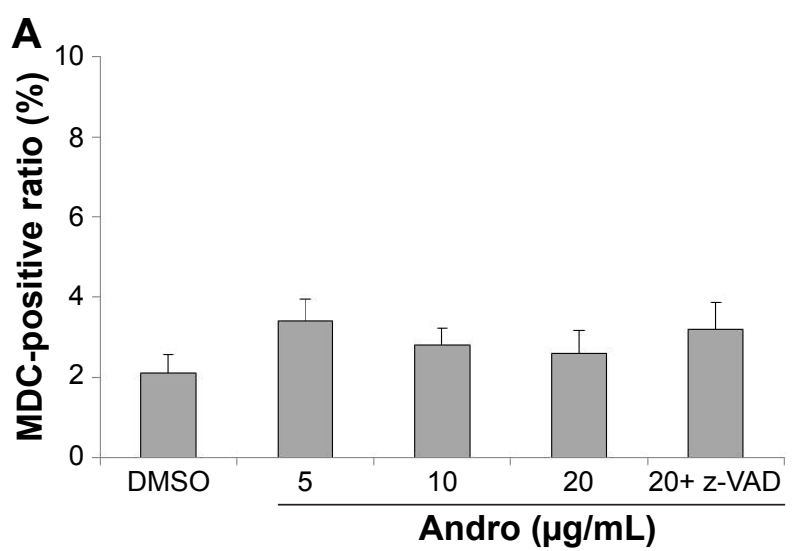

B

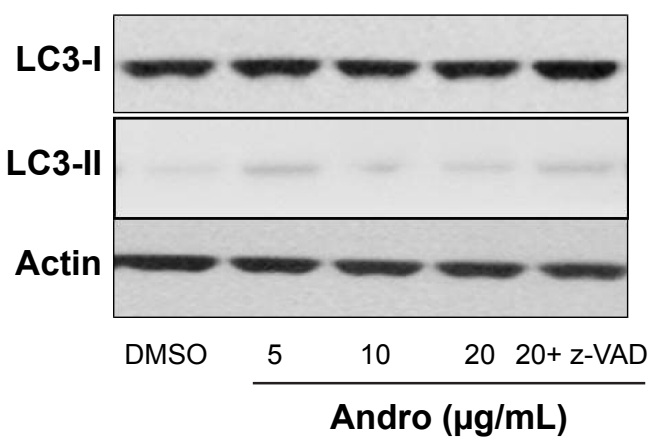

Figure 3 Andro could not induce autophagy in Jurkat cells.

Notes: (A) Jurkat cells were treated with different concentrations of Andro and subjected to quantitative analysis, detecting a positive ratio of MDC staining by flow cytometry. (B) Jurkat cells were washed with IX PBS and harvested on ice. Lysates were prepared in a lysis buffer, and then subjected to Western blot analysis to detect LC3 protein expression. Mean \pm SD, $n=3$.

Abbreviations: MDC, monodansylcadaverine; DMSO, dimethyl sulfoxide; Andro, Andrographolide; PBS, phosphate-buffered saline; SD, standard deviation.

A

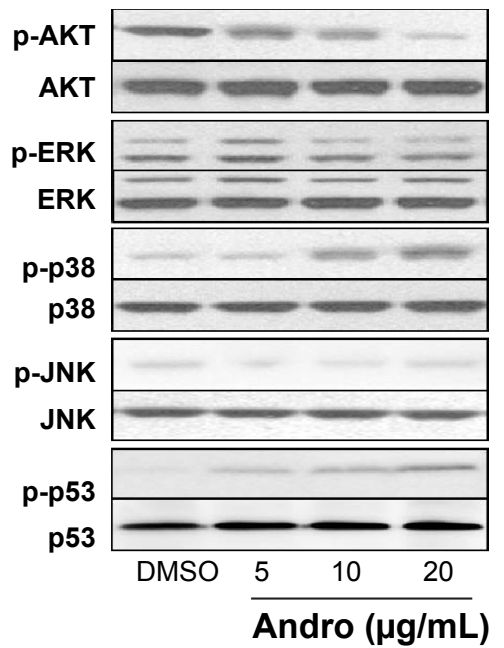

B

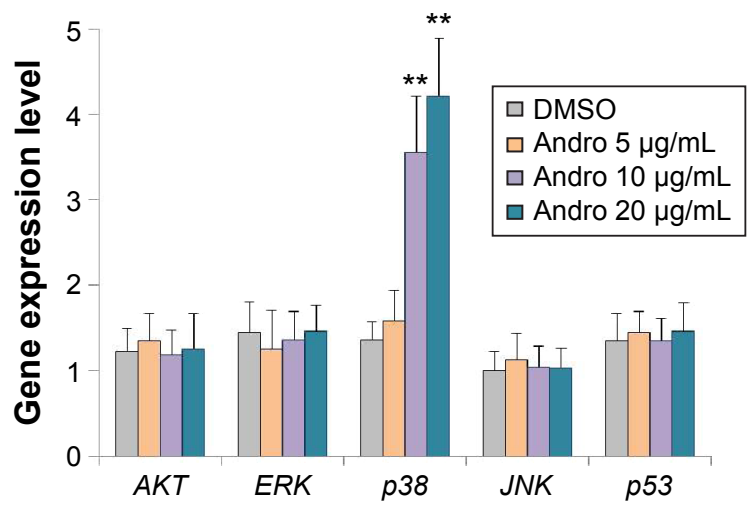

D

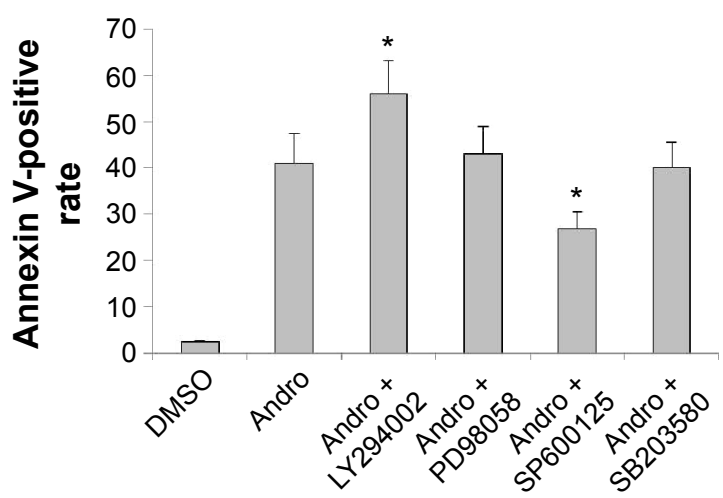

Figure 4 The effects of Andro on PI3K/AKT and MAPK pathways in Jurkat cells.

Notes: (A) Jurkat cells were treated with different concentrations of Andro for 48 hours. Later, the cells were washed with IX PBS and harvested on ice. Lysates were prepared in a lysis buffer, and then subjected to Western blot analysis to detect p-AKT, P-JNK, p-ERK, p-p38, and p-p53 proteins expression. (B) The cells were harvested and RNA was extracted from cell lysates using Qiagen RNA easy kits; RT-PCR was applied to detect AKT, p38, JNK, ERK RNA level. The target genes' expression levels were normalized to negative control GAPHD. Mean \pm SD, $n=3$. (C and D) The cells were pretreated with PD98059, SP600I25, or SB203580 for 0.5 hours, and then cultured with $20 \mu \mathrm{g} / \mathrm{mL}$ Andro for 48 hours. Later, the cells were harvested for MTT and Annexin V-FITC/PI double staining. $* P<0.05$, $* * P<0.0 \mathrm{I}$ versus DMSO group.

Abbreviations: Andro, Andrographolide; DMSO, dimethyl sulfoxide; PBS, phosphate-buffered saline; RT-PCR, real-time polymerase chain reaction; FITC, fluorescein isothiocyanate; PI, propidium iodide; SD, standard deviation; MTT, 3-(4,5-dimethyl-2-thiazolyl)-2,5-diphenyl-2-H-tetrazolium bromide. 


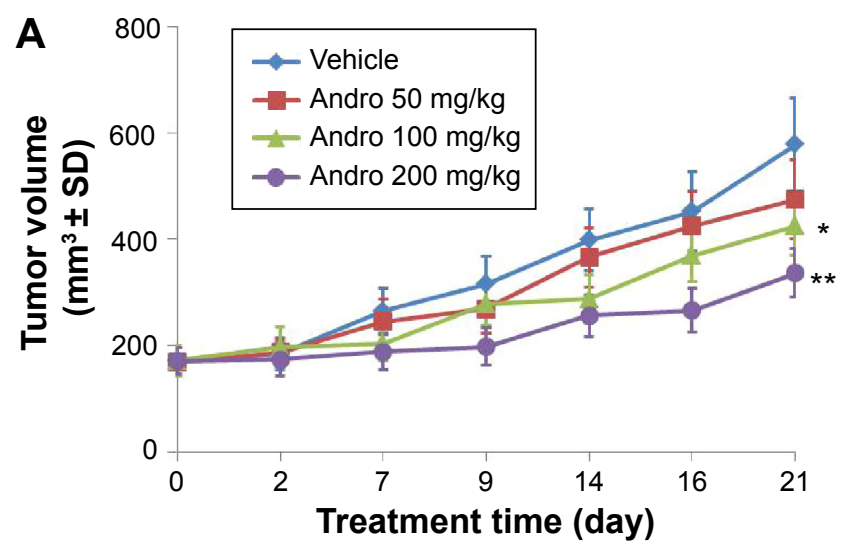

B

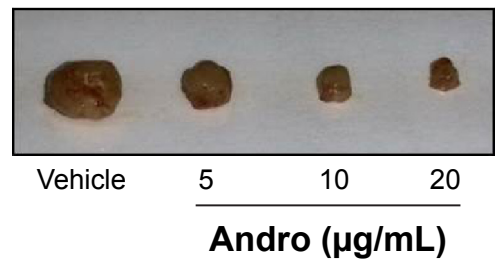

C

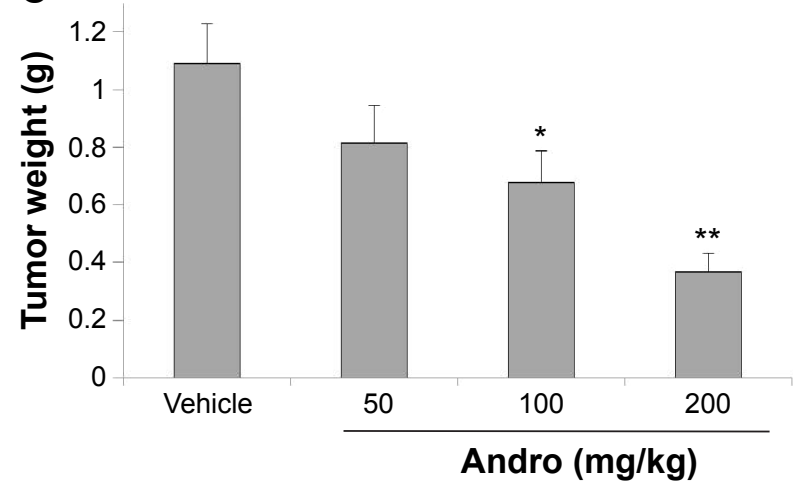

Figure 5 Antitumor effect of Andro on subcutaneous Jurkat xenografts.

Notes: (A) Nude mice bearing Jurkat tumors were randomly separated into four groups: vehicle, 50, 100, and $200 \mathrm{mg} / \mathrm{kg}$ Andro. Tumor volumes of mice were monitored twice a week. (B and $\mathbf{C}$ ) The mice were subjected to euthanasia and the tumors were collected and weighed after 3 weeks' treatment. $* P<0.05$ versus Andro group, $* * P<0.0$ I versus vehicle group.

Abbreviations: Andro, Andrographolide; SD, standard deviation.

in Jurkat cells. These data are in accordance with the results of the apoptosis assay (Figure 4D).

\section{Andro inhibited Jurkat xenografts tumor growth in vivo}

In view of the in vitro inhibitory effects of Andro on Jurkat cells, we next investigated the in vivo inhibitory action of Andro using nude mice. The mice were randomized into four groups: vehicle, 50,100, and $200 \mathrm{mg} / \mathrm{kg}$ Andro. The average tumor volume and average body weight were well balanced between each group. As we expected, Andro dose dependently inhibited Jurkat xenograft tumor growth after 3 weeks' treatment (Figure 5A). At the end of the study, the tumors were isolated from mice and weighted (Figure 5B and C). These in vivo findings further demonstrated that Andro significantly inhibited the growth of Jurkat xenografts.

\section{Discussion}

T-ALL is an acute form of leukemia, or cancer of the white blood cells, characterized by the overproduction and accumulation of cancerous, immature white blood cells. Even if an effective chemotherapeutic treatment was developed to treat T-ALL, the prognosis of it is still poor. Patients with T-ALL always acquired therapeutic resistance and tumor recurrence after standard treatment. ${ }^{23,24}$ Therefore, it is extremely necessary to develop more effective therapies to treat T-ALL patients.

Andro, the main constituent of A. paniculata, has been shown to possess many pharmacological effects on various cancers. For example, Andro induced human leukemia HL-60 cell apoptosis, which was due to an increase of the expression level of Bax. ${ }^{25}$ Similar to these results, Andro also induced T-ALL Jurkat cell apoptosis, which was confirmed when the pan-caspase inhibitor z-VAD was used in this study. In addition, Andro was reported to trigger autophagy instead of apoptosis in some type of cancer cells such as Huh-7, A549 cells. ${ }^{26,27}$ Besides, Andro was found to induce autophagic cell death in human liver cancer cells through cyclophilin D-mediated mitochondrial permeability transition pore. ${ }^{17}$ However, it is important to point out that 
no autophagosome was found when we treated Jurkat cells with different concentrations of Andro. It is possible that Andro may only induce autophagy in some specific tumor cell type.

As we know, the mitogen-activated protein kinase (MAPK) cascades could be activated by various cellular stresses and growth factors and are major signaling transduction molecules in apoptosis. ${ }^{28}$ MAPK signaling pathways including ERK, p38MAPK (p38), and JNK have been identified as chemotherapeutic targets for sensitizing cancer cells to apoptosis. ${ }^{29}$ Herein, we showed that inhibition of p38 activity clearly reduced Andro-induced apoptosis in Jurkat cells. In addition, the p-p38 protein expression and $p 38$ RNA level were increased after Andro treatments. These results suggest that p38 pathway was involved in Andro-induced apoptosis. Our data correlate with those previous findings, which indicated $\mathrm{p} 38$ played an important role in Andro-induced cell death. ${ }^{30,31}$ Yang et al $^{32}$ reported that Andro induces apoptosis of C6 glioma cells via the ERK/p53/caspase 7/PARP signaling pathway. However, Andro had no effect on JNK and ERK pathway in this study. Besides MAPK pathways, the PI3K/AKT signal transduction pathway plays a pivotal role in cell survival and prevents cancer cells from apoptosis during tumorigenesis. ${ }^{33}$ Our data show that Andro exerted a significant inhibitory effect on $\mathrm{p}$-AKT protein expression and that AKT-selective inhibitor could enhance Andro-induced apoptosis in Jurkat cells. These results suggest that PI3K/AKT pathway was involved in apoptosis.

Yang et $\mathrm{a}^{32}$ also mentioned that reactive oxygen species (ROS) are involved in Andro-induced C6 cell death. Nevertheless, there was no ROS generation in this study. The inconsistency may be due to different tumor types (Figure S1). It has become clear that the $\mathrm{p} 53$ protein interacts functionally with the MAPK pathways. When exposing cells to the stress, MAPK phosphorylates and then activates p53, which leads to cellular responses. ${ }^{34}$ Our data correlate with these previous findings. We found that when cells were treated with Andro, phosphorylation of p38 was significantly activated, and this phosphorylation then activated $\mathrm{p} 53$. Taken together, these data suggest that Andro might be a multitargeted inhibitor that performs its functions in a cell type-specific manner. Nevertheless, the detailed interaction between PI3K/AKT, p53, and p38 pathways is still unclear and further investigations are needed.

In conclusion, our findings unveil a novel mechanism of drug action by Andro in T-ALL cancer cells and suggest that Andro may induce T-ALL Jurkat cells death through AKT-p38MAPK-p53-caspase 3 signaling pathway. The successful application of Andro in an animal model represents a promising novel agent in the treatment of T-ALL cancer.

\section{Disclosure}

The authors report no conflicts of interest in this work.

\section{References}

1. Faderl S, O'Brien S, Pui CH, et al. Adult acute lymphoblasticleukemia: concepts and strategies. Cancer. 2010;116(5):1165-1176.

2. Pui CH. Acute lymphoblastic leukemia. Introduction. Semin Hematol. 2009;46(1):1-2.

3. Pui CH, Relling MV, Downing JR. Acute lymphoblastic leukemia. N Engl J Med. 2004;350(15):1535-1548.

4. Vivanco I, Sawyers CL. The phosphatidylinositol 3-kinase AKT pathway in human cancer. Nat Rev Cancer. 2002;2(7):489-501.

5. Engelman JA, Luo J, Cantley LC. The evolution of phosphatidylinositol 3-kinases as regulators of growth and metabolism. Nat Rev Genet 2006;7(8):606-619.

6. Courtney KD, Corcoran RB, Engelman JA. The PI3K pathway as drug target in human cancer. J Clin Oncol. 2010;28(6):1075-1083.

7. Sharma A, Lal K, Handa SS. Standardization of the Indian crude drug kalmegh by high-pressure liquid-chromatographic determination of andrographolide. Phytochem Anal. 1992;3(3):129-131.

8. Nanduri S, Nyavanandi VK, Thunuguntla SSR, et al. Synthesis and structure-activity relationships of andrographolide analogues as novel cytotoxic agents. Bioorg Med Chem Lett. 2004;14(18):4711-4717.

9. Zhao F, He EQ, Wang L, Liu K. Anti-tumor activities of andrographolide, a diterpene from Andrographis paniculata, by inducing apoptosis and inhibiting VEGF level. J Asian Nat Prod Res. 2008;10(5):467-473.

10. Naik SR, Hule A. Evaluation of immunomodulatory activity of an extract of andrographolides from Andographis paniculata. Planta Med. 2009;75(8):785-791.

11. Wiart C, Kumar K, Yusof MY, Hamimah H, Fauzi ZM, Sulaiman M. Antiviral properties of ent-labdene diterpenes of Andrographis paniculata Nees, inhibitors of herpes simplex virus type 1. Phytother Res. 2005;19(12):1069-1070.

12. Tsai HR, Yang LM, Tsai WJ, Chiou WF. Andrographolide acts through inhibition of ERK1/2 andAkt phosphorylation to suppress chemotactic migration. Eur J Pharmacol. 2004;498(1-3):45-52.

13. Sukumari-Ramesh S, Bentley JN, Laird MD, Singh N, Vender JR, Dhandapani KM. Dietary phytochemicals induce p53- and caspaseindependent cell death in human neuroblastoma cells. Int J Dev Neurosci. 2011;29(7):701-710.

14. Shi MD1, Lin HH, Chiang TA, Tsai LY, Tsai SM, Lee YC. Andrographolide could inhibit human colorectal carcinoma Lovo cells migration and invasion via down-regulation of MMP-7 expression. Chem Biol Interact. 2009;180(3):344-352.

15. Cheung HY, Cheung SH, Li J, et al. Andrographolide isolated from Andrographis paniculata induces cell cycle arrest and mitochondrialmediated apoptosis in human leukemic HL-60 cells. Planta Med. 2005; 71(2):1106-1111.

16. Chen W, Feng L, Nie H, Zheng X. Andrographolide induces autophagic cell death in human liver cancer cells through cyclophilin D-mediated mitochondrial permeability transition pore. Carcinogenesis. 2012;33: 2190-2198.

17. Luo GX, Cai J, Lin JZ, et al. Autophagy inhibition promotes gambogic acid-induced suppression of growth and apoptosis in glioblastoma cells Asian Pac J Cancer Prev. 2012;13(13):6211-6216.

18. Wong HC, Wong CC, Sagineedu SR, Loke SC, Lajis NH, Stanslas J. SRJ23, a new semisynthetic andrographolide derivative: in vitro growth inhibition and mechanisms of cell cycle arrest and apoptosis in prostate cancer cells. Cell Biol Toxicol. 2014;30(5):269-288. 
19. Yang S, Evens AM, Prachand S, et al. Mitochondrial-mediated apoptosis in lymphoma cells by the diterpenoid lactone andrographolide, the active component of Andrographis paniculata. Clin Cancer Res. 2010; 16(19):4755-4768.

20. Higgins GC, Devenish RJ, Beart PM, Nagley P. Autophagic activity in cortical neurons under acute oxidative stress directly contributes to cell death. Cell Mol Life Sci. 2011;68(22):3725-3740.

21. Li J, Zhang C, Jiang H, Cheng J. Andrographolide inhibits hypoxiainducible factor-1 through phosphatidylinositol 3-kinase/AKT pathway and suppresses breast cancer growth. Onco Targets Ther. 2015; 8:427-435.

22. Gong C, Xu C, Ji L, Wang Z. A novel semi-synthetic andrographolide analogue A5 inhibits tumor angiogenesis via blocking the VEGFR2p38/ERK1/2 signal pathway. Biosci Trends. 2013;7(5):230-236.

23. Bassan R, Hoelzer D. Modern therapy of acute lymphoblastic leukemia. J Clin Oncol. 2011;29(5):532-543.

24. Gómez AM, Martínez C, González M, et al. Chemokines and relapses in childhood acute lymphoblastic leukemia: a role in migration and in resistance to antileukemic drugs. Blood Cells Mol Dis. 2015;55(3):220-227.

25. Manikam SD, Stanslas J. Andrographolide inhibits growth of acute promyelocytic leukaemia cells by inducing retinoic acid receptorindependent cell differentiation and apoptosis. J Pharm Pharmacol. 2009;61(1):69-78.

26. Zhou J, Zhang S, Ong CN, Shen HM. Critical role of pro-apoptotic Bcl-2 family members in andrographolide-induced apoptosis in human cancer cells. Biochem Pharmacol. 2006;72(2):132-144.
27. Lee YC, Lin HH, Hsu CH, Wang CJ, Chiang TA, Chen JH. Inhibitory effects of andrographolide on migration and invasion in human nonsmall cell lung cancer A549 cells via down-regulation of PI3K/Akt signaling pathway. Eur J Pharmacol. 2010;632(1-3):23-32.

28. Qi MS, Elion EA. MAP kinase pathways. J Cell Sci. 2005;118(16): 3569-3572.

29. Su CC, Chen JY, Din ZH, et al. 13-acetoxysarcocrassolide induces apoptosis on human gastric carcinoma cells through mitochondriarelated apoptotic pathways: p38/JNK activation and PI3K/AKT suppression. Mar Drugs. 2014;12(10):5295-5315.

30. Pratheeshkumar P, Kuttan G. Andrographolide inhibits human umbilical vein endothelial cell invasion and migration by regulating MMP-2 and MMP-9 during angiogenesis. J Environ Pathol Toxicol Oncol. 2011; 30(1):33-41.

31. Chen YY, Hsieh CY, Jayakumar T, et al. Andrographolide induces vascular smooth muscle cell apoptosis through a SHP-1-PP2Ap38MAPK-p53 cascade. Sci Rep. 2014;4:5651.

32. Yang SH, Wang SM, Syu JP, et al. Andrographolide induces apoptosis of C6 glioma cells via the ERK-p53-caspase 7-PARP pathway. Biomed Res Int. 2014;(7):871-882.

33. Castaneda CA, Cortes-Funes H, Gomez HL, Ciruelos EM. The phosphatidyl inositol 3-kinase/AKT signaling pathway in breast cancer. Cancer Metastasis Rev. 2010;29(4):751-759.

34. Pan ST, Qin Y, Zhou ZW, et al. Plumbagin induces G2/M arrest, apoptosis, and autophagy via p38 MAPK- and PI3K/Akt/mTORmediated pathways in human tongue squamous cell carcinoma cells. Drug Des Devel Ther. 2015;9:1601-1626. 


\section{Supplementary material}

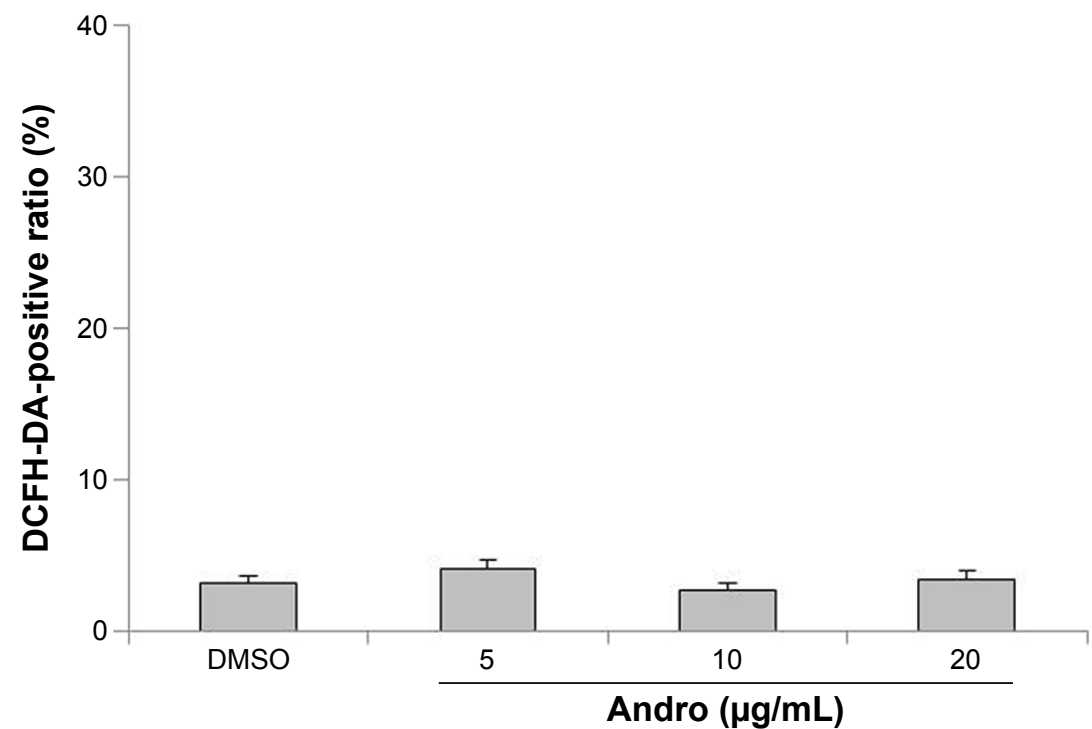

Figure SI Andro could not induce ROS generation in Jurkat cells.

Notes: Jurkat cells were treated with different concentrations of Andro and subjected to quantitative analysis, detecting a positive ratio of DCFH-DA staining by flowcytometry. DCFH-DA is a specific marker for ROS detection.

Abbreviations: DCFH-DA, 2',7'-dichlorofluorescin diacetate; DMSO, dimethyl sulfoxide; Andro, Andrographolide; ROS, reactive oxygen species.

\section{Publish your work in this journal}

Drug Design, Development and Therapy is an international, peerreviewed open-access journal that spans the spectrum of drug design and development through to clinical applications. Clinical outcomes, patient safety, and programs for the development and effective, safe, and sustained use of medicines are a feature of the journal, which has also been accepted for indexing on PubMed Central. The manuscript management system is completely online and includes a very quick and fair peer-review system, which is all easy to use. Visit http://www.dovepress.com/testimonials.php to read real quotes from published authors.

Submit your manuscript here: http://www.dovepress.com/drug-design-development-and-therapy-journal 